\title{
Tighter Throughput Lower Bounds of Connectivity-Based Reliable Multicast MAC Protocol for IEEE 802.11 Wireless LANs
}

\author{
Woo-Yong Choi ${ }^{\dagger}$ \\ Department of Industrial and Management Systems Engineering, Dong-A University \\ IEEE 802.11 무선랜에서 연결정보 기반의 신뢰성 있는
멀티캐스트 MAC 프로토콜을 위한 쓰루풋의 하한 유도 \\ 최 우 용 \\ 동아대학교 산업경영공학과
}

\begin{abstract}
The connectivity-based reliable multicast MAC protocol was proposed for IEEE 802.11 wireless LANs and the formulae for the lower bounds of the multicast downlink throughput and the uplink throughput of the connectivitybased reliable multicast MAC protocol were derived in our previous study. We provide the tighter throughput lower bounds of the connectivity-based reliable multicast MAC protocol than the lower bounds presented in our previous study.
\end{abstract}

Keywords: IEEE 802.11 Wireless LAN, Reliable Multicast, Throughput

\section{Introduction}

To satisfy the demands for high rate wireless data service with the QoS (Quality of Service) provision, various versions of IEEE 802.11 standards have been developed to support IEEE 802.11, IEEE 802.11a, IEEE 802.11b, IEEE 802.11g, IEEE 802.11e and IEEE 802.11n wireless LANs (IEEE, 2003; IEEE Std 802.11g, 2003; IEEE Std 802.11e, 2005; IEEE Std $802.11 \mathrm{n}, 2009$ ). After the data frames are transmitted, the transmitters wait for the ACK frames from the recipients. The transmitters regard the data frame transmissions to be successful only when the corresponding ACK frames are received successfully. If the ACK frames are not received successfully, the corresponding data frames are retransmitted. The reliable unicast data transmission service is provided by this retransmission method in wireless LANs. However, the reliable multicast data transmission service has not been realized yet in wireless LANs. In the literature, the BMMM (Batch Mode Multicast MAC) protocol and the connectivitybased reliable multicast MAC protocol are proposed in Sun, Huang et al. (2003), Choi (2009), Choi (2010A), and Choi (2010B). The connectivity-based reliable multicast MAC protocol enhances the BMMM protocol by reducing the necessary control frame transmissions significantly.

To derive the lower bounds of the multicast downlink throughput and the uplink throughput of the connectivitybased reliable multicast MAC protocol in Choi (2009), and Choi (2010A), it was assumed that the mean number of connected polling sequences for each retransmission of the multicast data frame equals to the mean number of connected polling sequences for the initial transmission of the multicast data frame. This assumption can lead to the inaccurate throughput estimation especially when there are many multi-

\footnotetext{
This study was supported by research funds from Dong-A University.

† Corresponding author : Professor Woo-Yong Choi, Department of Industrial and Management Systems Engineering, Dong-A University, 840 Hadan-2-dong, Saha-gu, Busan, 604-714, Korea, Fax :+82-51-200-7697, E-mail : wychoi77@dau.ac.kr Received October 5, 2012; Revision Received October 22, 2012; Accepted October 23, 2012.
} 
cast recipient nodes in IEEE 802.11 wireless LANs or the wireless channel is not good. For the explicit formulae for the throughput lower bounds, one can refer to Equations (5) (8) in Choi (2009) and Equation (1) in Choi (2010A).

In this paper, relaxing the assumption, we provide the tighter throughput lower bounds of the connectivity-based reliable multicast MAC protocol. By numerical examples, we show that the throughput lower bounds are improved.

This paper is organized as follows. In the next section, we provide the tighter throughput lower bounds of the connectivitybased reliable multicast MAC protocol than the lower bounds presented in Choi (2009), and Choi (2010A). In Section 3, numerical examples are presented to show the improvement by the new throughput lower bounds. Finally, conclusions are presented in Section 4.

\section{Tighter Throughput Lower Bounds}

$n$ represents the number of recipient nodes of the initial multicast data transmission. Let us denote by $N_{R A K}(v)$ the mean number of connected polling sequences for the initial transmission or the retransmission of the multicast data frame when the multicast data frame is transmitted to $v$ recipients, and by $T(v)$ the mean amount of time taken for the multicast data frame to be successfully transmitted to $v$ recipients. Then, to relax the assumption explained in Section 1 and obtain the tighter upper bounds $T^{\mathrm{U}}(v)$ of $T(v)$ for $v=1,2, \cdots, n$, we can modify Equation (6) in Choi (2009) and Equation (1) in Choi (2010A) and derive the following linear equations with respect to the unknowns $T^{\mathrm{U}}(v)$ for $v=1,2, \cdots, n$ :

$$
\begin{aligned}
& T^{U}(\nu)=\left(\mathrm{SIFS}+T_{M}\right)+\nu \cdot\left(\frac{1-p}{1-p_{\mathrm{ACK}}}\right) \\
&\left(\mathrm{SIFS}+\mathrm{T}_{A C K}+\frac{q \cdot L_{U}}{R}\right) \\
&+ N_{\mathrm{RAK}}(\nu) \cdot\left(\mathrm{SIFS}+T_{\mathrm{RAK}}+\frac{48 \cdot\left(2 \cdot \nu / N_{\mathrm{RAK}}(\nu)-1\right.}{R}\right) \\
&+ \nu \cdot p \cdot\left(\Pi \mathrm{FS}+T_{\mathrm{RAK}}+\frac{48 \cdot\left(2 \cdot \nu / N_{\mathrm{RAK}}(\nu)-1\right.}{R}\right) \\
&+ \sum_{t=1}^{\nu}\left(\begin{array}{l}
\nu \\
t
\end{array}\right) p^{t} \cdot(1-p)^{\nu-t} \cdot T^{U}(t) \\
&+(1-p)^{\nu} \cdot\left(\mathrm{SIFS}+T_{R A K}+\frac{\nu}{N_{\mathrm{RAK}}(\nu) \cdot R}\right) \\
& \text { for } \nu=1,2, \cdots, n .
\end{aligned}
$$

For the definitions of SIFS, $T_{M}, p, p_{\mathrm{ACK}}, T_{\mathrm{ACK}}, q, R, L_{U}$, $T_{R A K}$, and PIFS, we can refer to Choi (2009), and Choi (2010A). The first term, the second term, and the third and the fourth terms in (1), respectively, take into account the amount of time taken to transmit the multicast data frame, the ACK frames, and the RAK polling frames until the first RAK polling procedures for the connected polling sequences for $v$ recipients are completed. The fourth term is due to that $\nu \cdot p$ is the mean number of error occurrences during the handshakes of the multicast data frame and the corresponding ACK frames, and the AP should wait for PIFS to transmit the next RAK polling frames when the handshakes fail. The fifth term takes into account the amount of time taken for the multicast data frame to be successfully retransmitted to one or more failed recipients when the errors occur during the handshakes of the multicast data frame and the corresponding ACK frames. The last term takes into account the amount of time taken to transmit the group ACK frame when no error occurs during the handshakes of the multicast data frame and the corresponding ACK frames.

From (1), we can see that $T^{\mathrm{U}}(1)$ can be obtained by the equation for $v=1, T^{\mathrm{U}}(2)$ can be obtained by the equation for $v=2$, and $T^{\mathrm{U}}(1)$, and $T^{\mathrm{U}}(3)$ can be obtained by the equation for $v=3$, and $T^{\mathrm{U}}(1)$ and $T^{\mathrm{U}}(2)$. In this manner, we can obtain all $T^{\mathrm{U}}(v)$ for $v=1,2, \cdots, n$ by the equations in (1). Finally, like Equations (7) and (8) in Choi (2009) we can obtain the lower bounds of the multicast downlink throughput $E_{\mathrm{D}}$ and the uplink throughput $E_{\mathrm{U}}$ as follows :

$$
\begin{aligned}
& E_{D} \geq \frac{n \cdot L_{D}}{T^{U}(n)} \\
& E_{U} \geq \frac{(1-p) \cdot q \cdot n \cdot E\left[X_{i}\right] \cdot L_{U}}{T^{U}(n)}
\end{aligned}
$$

\section{Numerical Results}

Using (1), (2) and (3), we calculated the lower bounds of the multicast downlink throughputs of the connectivity-based reliable multicast MAC protocol for the values of the parameters in $<$ Table 2> in Choi (2009). The cases of the probability $p$ of error during the handshake equal to $10 \%$ and $15 \%$ were additionally considered. In $\langle$ Figure 1$\rangle$ and $\langle$ Figure 2$\rangle$, we compare the numerical results of the lower bounds of the multicast downlink throughputs of the connectivity-based reliable multicast MAC protocol that are proposed in this paper and Choi (2009) and Choi (2010A), and the multicast downlink throughput of the BMMM protocol that is proposed in Choi (2009) and Choi (2010A).

Trivially $N_{R A K}(1)=1$. The estimates of $N_{R A K}(v)$ for $v=3,5$, $10,20, \cdots, 100$ can be found in $<$ Table $1>$. To calculate the throughput lower bounds using (1) and (2), we need to estimate $N_{R A K}(v)$ for $v=1,2, \cdots, 100$. If $v_{1}<v_{2}$ and the estimates of $N_{R A K}\left(v_{1}\right)$ and $N_{R A K}\left(v_{2}\right)$ are consecutively found in <Table 1>, all missing $N_{R A K}\left(v_{1}+1\right), N_{R A K}\left(v_{1}+2\right), \cdots, N_{R A K}\left(v_{2}-1\right)$ 
were approximated by $N_{R A K}\left(v_{2}\right)$. Generally as $v$ increases, $N_{R A K}(v)$ increases. Therefore, (1) and (2) still provide the throughput lower bounds under this approximation. Table 1 includes the experimental results in $<$ Table $1>$ in Choi (2009) and the results obtained by the additional experiment using the dynamic binary search algorithm and the simulation method explained in Choi (2009).

Table 1. Results of simulation experiment

\begin{tabular}{c|c|c}
\hline$v$ & $N_{R A K}(v)$ & Mean Necessary Time $(\mu s)$ \\
\hline 3 & 1.7 & 1 \\
\hline 5 & 1.6 & 1 \\
\hline 10 & 1 & 1 \\
\hline 20 & 1.1 & 1.5 \\
\hline 30 & 2.1 & 2.1 \\
\hline 40 & 1 & 1 \\
\hline 50 & 4 & 3 \\
\hline 60 & 5.1 & 2.3 \\
\hline 70 & 7.9 & 2.9 \\
\hline 80 & 5 & 3.1 \\
\hline 90 & 7.2 & 2.1 \\
\hline 100 & 12.1 & 3.9 \\
\hline
\end{tabular}

Since we set the probability $q$ to 1 , that is, all ACK frames have the piggybacked uplink data frames, the uplink throughput results of the BMMM and the connectivity-based reliable multicast MAC protocols were, respectively, the same as the multicast downlink throughput results of the BMMM and the connectivity-based reliable multicast MAC protocols. From $<$ Figure $1>$ and $<$ Figure $2>$, we can see that the throughput lower bounds proposed in this paper are greater than the throughput lower bounds proposed in Choi (2009) and Choi (2010A) in all cases. The gap between the throughput lower bounds proposed in this paper and Choi (2009) and Choi (2010A) becomes greater as $n$ increases and $p$ becomes larger. This is because that the effect of the assumption explained in Section 1 on the estimation of the MAC throughput becomes greater as $n$ increases and $p$ becomes larger.

When $n=100$ and $p=1 \%$, the throughput lower bounds proposed in this paper are greater than the throughput lower bounds proposed in Choi (2009) and Choi (2010A) by about 8 $\%$ and $6 \%$ with the user payloads of 88 bits and 1000 bits, respectively. When $n=100$ and $p=5 \%$, the new throughput lower bounds are greater than the throughput lower bounds proposed in Choi (2009) and Choi (2010A) by about 13\% and $10 \%$ with the user payloads of 88 bits and 1000 bits, respectively. When $n=100$ and $p=15 \%$, the new throughput lower bounds are greater than the throughput lower bounds proposed in Choi (2009) and Choi (2010A) by about $20 \%$ and $16 \%$ with the user payloads of 88 bits and 1000 bits, respectively.

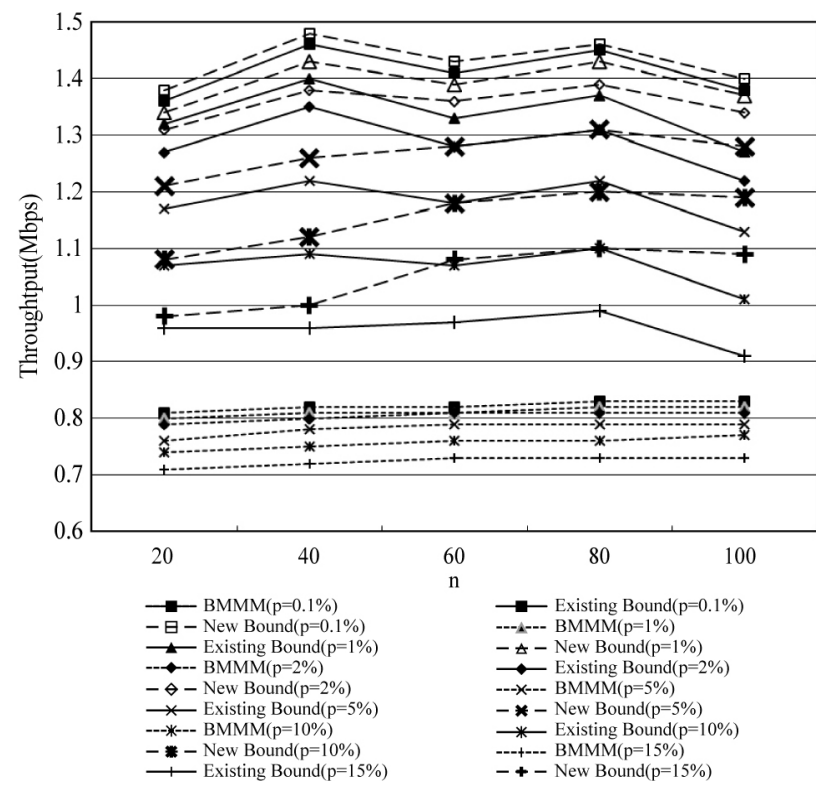

Figure 1. Throughput Results with multicast and Uplink user payloads of 88 bits

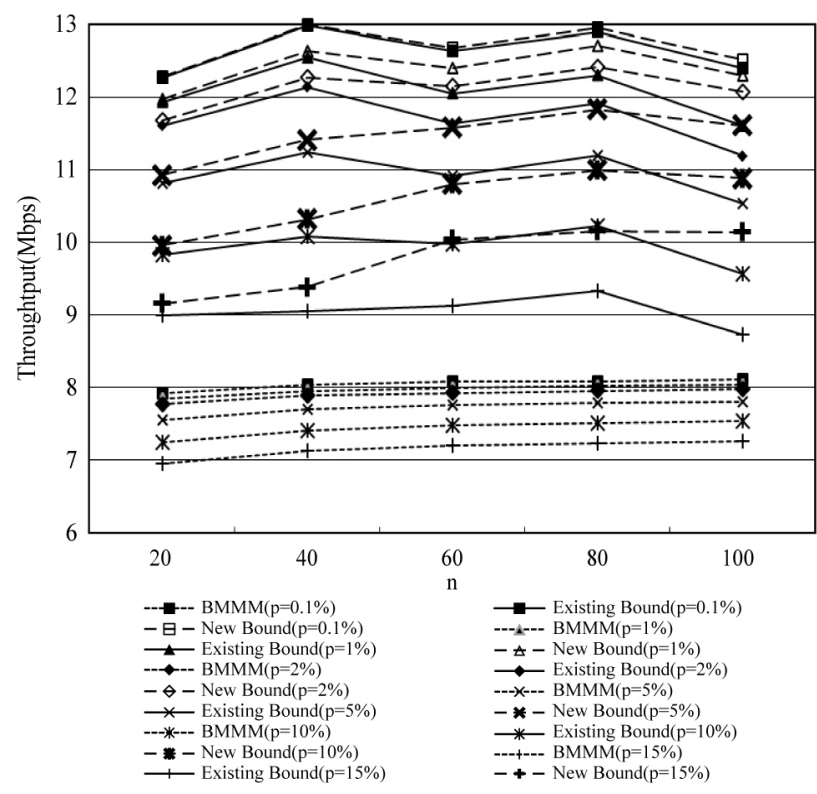

Figure 2. Throughput Results with multicast and uplink user payloads of 1000 bits

\section{Conclusions}

In this paper, we provided the tighter throughput lower bounds of the connectivity-based reliable multicast MAC protocol. By numerical examples, we showed that the throughput lower bounds are improved in all cases and the improvement becomes prominent as the number of multicast recipient nodes increases and the wireless channel gets worse. 


\section{References}

Choi, W.-Y. (2009), Connectivity-Based Reliable Multicast MAC Protocol for IEEE 802.11 Wireless LANs, EURASIP Journal on Wireless Communications and Networking, 2009.

Choi, W.-Y. (2010A), Corrections to Connectivity-Based Reliable Multicast MAC Protocol for IEEE 802.11 Wireless LANs, EURASIP Journal on Wireless Communications and Networking, 2010.

Choi, W.-Y. (2010B), Improved Connectivity-Based Reliable Multicast MAC Protocol for IEEE 802.11 Wireless LANs, Journal of Korean Institute of Industrial Engineers, 36(2), 94-100.
IEEE (2003), IEEE Wireless LAN Edition, IEEE Press.

IEEE Std 802.11g (2003), Wireless LAN Medium Access Control (MAC) and Physical Layer Specifications: Higher Speed Physical Layer (PHY) Extension in the $2.4 \mathrm{GHz}$ Band.

IEEE Std 802.11e (2005), Wireless LAN Medium Access Control (MAC) and Physical Layer Specifications : Medium Access Control (MAC) Quality of Service (QoS) Enhancements.

IEEE Std 802.11n (2009), Wireless LAN Medium Access Control (MAC) and Physical Layer (PHY) Specifications : Enhancements for Higher Throughput.

Sun, M., Huang, L., Wang, S., Arora, A., and Lai, T. (2003), Reliable MAC Layer Multicast in IEEE 802.11 Wireless Networks, Wireless Communications and Mobile Computing, 3(4), 439-453. 